\title{
Role of Radical Formation at Tyrosine 193 in the Allene Oxide Synthase Domain of a Lipoxygenase-AOS Fusion Protein from Coral ${ }^{\dagger}$
}

\author{
Fayi Wu, ${ }^{\ddagger}$ Leron J. Katsir, ${ }^{\ddagger}$ Margaret Seavy, and Betty J. Gaffney* \\ Department of Biological Science, Florida State University, Tallahassee, Florida 32306-4370 \\ Received December 30, 2002; Revised Manuscript Received April 3, 2003
}

\begin{abstract}
Coral allene oxide synthase (cAOS), a fusion protein with $8 R$-lipoxygenase in Plexaura homomalla, is a hemoprotein with sequence similarity to catalases. cAOS reacts rapidly with the oxidant peracetic acid to form heme compound I and intermediate II. Concomitantly, an electron paramagnetic resonance (EPR) signal with tyrosyl radical-like features, centered at a $g$-value of 2.004-2.005, is formed. The radical is identified as tyrosyl by changes in EPR spectra when deuterated tyrosine is incorporated in cAOS. The radical location in cAOS is determined by mutagenesis of Y193 and Y209. Upon oxidation, native cAOS and mutant Y209F exhibit the same radical spectrum, but no significant tyrosine radical forms in mutant Y193H, implicating Y193 as the radical site in native cAOS. Estimates of the side chain torsion angles for the radical at Y193, based on the $\beta$-proton isotropic EPR hyperfine splitting, $A_{\text {iso }}$, are $\theta_{1}=21$ to $30^{\circ}$ and $\theta_{2}=-99$ to $-90^{\circ}$. The results show that cAOS can cleave nonsubstrate hydroperoxides by a heterolytic path, although a homolytic course is likely taken in converting the normal substrate, $8 R$-hydroperoxyeicosatetraenoic acid $(8 R$-HpETE), to product. Coral AOS achieves specificity for the allene oxide formed by selection of the homolytic pathway normally, while it inactivates by the heterolytic path with nonoptimal substrates. Accordingly, with the nonoptimal substrate, $13 R$-hydroperoxyoctadecadienoic acid (13R-HpODE), mutant $\mathrm{Y} 193 \mathrm{H}$ is inactivated after turning over significantly fewer substrate molecules than required to inactivate native cAOS or the Y209F mutant because it cannot absorb oxidizing equivalents by forming a radical at Y193.
\end{abstract}

Polyunsaturated fatty acids (PUFAs) ${ }^{1}$ are rapidly converted to cell signaling molecules in multistep syntheses involving an initial lipoxygenase or prostaglandin synthase step, followed by further enzymatic conversions $(1-3)$. These multistep syntheses are regulated by localization of the enzymes in subcellular compartments and by protein - protein associations. Examples include synthesis of leukotriene $\mathrm{C}_{4}$ at the nuclear envelope of leukocytes (4) and synthesis of jasmonic acid by steps which are thought to begin in chloroplasts and to be completed in peroxisomes of green plant cells (5). A fusion protein identified in the coral Plexaura homomalla represents a third mode of regulation, in which an $\mathrm{N}$-terminal catalase-like domain is fused to an $8 R$-lipoxygenase domain (6). The $\mathrm{N}$-terminal domain (44 $\mathrm{kDa}$ ) has allene oxide synthase (cAOS) activity with the $8 R$ -

$\dagger$ This research was supported by National Institutes of Health Grant GM36232 (to B.J.G.).

* To whom correspondence should be addressed. Phone: (850) 6448547. Fax: (850) 644-8547. E-mail: gaffney@ bio.fsu.edu.

$\doteqdot$ These authors contributed equally.

${ }^{1}$ Abbreviations: cAOS, coral allene oxide synthase; BLC, bovine liver catalase; BSA, bovine serum albumin; CYP, cytochrome P450; DES, divinyl ether synthase; EPR, electron paramagnetic resonance; $8 R$-HpETE, $8 R$-hydroperoxy- $9 E, 11 Z$-eicosatetraenoic acid; $13 R$-HpODE, $13 R$-hydroperoxy-9Z,11E-octadecadienoic acid; HPL, hydroperoxy lyase; HPLC, high-performance liquid chromatography; IPTG, isopropyl $\beta$-D-1-thiogalactopyranoside; LB, Luria broth; PAGE, polyacrylamide gel electrophoresis; PGHS, prostaglandin $\mathrm{H}$ synthase; $\mathrm{PGH}_{2}$, prostaglandin endoperoxide $\mathrm{H}_{2} ; \mathrm{PGI}_{2}$, prostacyclin; $\mathrm{PMC}$, Proteus mirabilis catalase; PUFA, polyunsaturated fatty acids; TB, terrific broth; Tris$\mathrm{HCl}$, tris(hydroxymethyl)aminomethane hydrochloride. hydroperoxide of arachidonic acid ( $8 R$-HpETE) in the intact fusion protein, as well as when it is expressed separately, but it does not exhibit catalase activity (7). Evidence from sequence and spectroscopy has confirmed that the similarity of coral AOS to catalase includes tyrosinate axial ligation to heme iron $(6,8)$. Gene fusion events in one organism often suggest functional association of separate proteins in other organisms (9). The fusion of $8 R$-lipoxygenase and catalaselike AOS domains in coral is the first instance of coupling lipoxygenase products to a second enzyme with a heme center in which the proximal ligand is tyrosine. This contrasts with known pathways in which lipoxygenase products are converted by the thiolate-ligated cytochrome P450s. To probe the significance of the coral fusion, reactions of the coral AOS domain with a pseudosubstrate of catalases have been examined.

The AOS from coral is not related in sequence (6) to the allene oxide synthases that are abundant in plants $(5,10-$ 12). Allene oxide synthase activity in plants is carried out by cytochrome P450 enzymes in the CYP74 group of atypical P450s (2). Enzymes in this family catalyze isomerizations of fatty acid hydroperoxides without requiring reductants or molecular oxygen $(2,9)$. Plant enzymes in the CYP74 family include allene oxide synthases $(5,11-12)$, hydroperoxylyases (HPL) $(5,12)$, and divinyl ether synthase (DES) (13). Animal P450s with catalytic requirements similar to those of the CYP74 enzymes include CYP5A1, thromboxane synthase $(14,15)$, which converts prostaglandin $\mathrm{H}_{2}$ $\left(\mathrm{PGH}_{2}\right)$ to thromboxane $\mathrm{A}_{2} ; \mathrm{CYP} 8$, prostacyclin synthase (15, 
16), which converts $\mathrm{PGH}_{2}$ to prostacyclin $\left(\mathrm{PGI}_{2}\right)$; and CYP2B1, which forms epoxy alcohol products by a mechanism thought to proceed through the same intermediates as those of the AOS reaction (17). The proposed mechanisms of these P450 enzymes and the proposed cAOS mechanism invoke homolysis of the $\mathrm{O}-\mathrm{O}$ bond by ferric iron to yield an $\mathrm{Fe}^{4+}-\mathrm{OH}$ intermediate and a protein-bound substrate radical $(7,17)$, followed by rapid product formation without substantial substrate reorientation (18). In known reactions of CYPs with synthetic hydroperoxides, the balance of heterolysis to homolysis of a particular hydroperoxide is delicately controlled by interactions of the oxidants with the protein structure (18).

It is interesting that coral AOS inactivates rapidly, after turning over only $\sim 32000$ molecules of substrate (7). Inactivation of cAOS could occur if occasional heterolytic cleavage of the hydroperoxide substrate results in overoxidation of the heme or the protein. In catalases, compound I is formed by heterolysis of hydrogen peroxide and a variety of substrate analogues, ROOH (19). In some catalases, the subsequent transfer of an electron from tyrosine to the porphyrin $\pi$-cation radical of compound I gives intermediate II, with an iron oxyferryl and a protein-bound tyrosine radical $(20-23)$. Since cAOS does not react readily with hydrogen peroxide, and it reacts very rapidly (1400 turnovers $\mathrm{s}^{-1}$ ) with its natural substrate, $8 R$-hydroperoxyeicosatetraenoic acid ( $8 R$-HpETE) (7), it has been of interest to examine whether there are reactions of an intermediate rate, with other hydroperoxides, that might allow cAOS intermediates to be trapped. We reported that cAOS, like bovine liver catalase (BLC), reacts with peracetic acid to form a protein radical with features in the EPR spectrum suggesting tyrosyl as the origin (8). Tyrosines 214, 235, and 369 in BLC have been discussed most often as possible sites for the radical formed in that enzyme $(21,22)$. Recently, the site of radical formation in mutant Proteus mirabilis catalase (PMC) was examined by biophysical techniques (23). Although PMC does form a radical when tyrosine is substituted for the native phenylalanine at position 194 (equivalent to Y193 in cAOS), it is argued $(21,23)$ that the BLC radical occurs differently at Y369. Coral AOS has four tyrosines in positions similar to BLC tyrosines: cAOS Y193, Y209, and Y269 and the probable heme ligand, Y353 (equivalent in BLC to Y214, Y230, Y324, and Y357, respectively), but a sequence similar to BLC Y369 and immediate neighbors does not occur in coral AOS (6). We have examined whether free radical formation in the coral AOS domain involves sequence regions in cAOS that correspond to sequences in catalases. These studies include optical detection of oxidized heme intermediates in cAOS, mutation at two tyrosine sites (Y193 and Y209), and EPR studies of the radicals formed in oxidized native and deuterium-substituted tyrosine cAOS. The results indicate that the mechanism of cAOS inactivation during turnover involves a significant number of heterolytic reactions with hydroperoxides bound nonoptimally in the substrate site.

\section{EXPERIMENTAL PROCEDURES}

Chemicals. Oligonucleotides were synthesized by Integrated DNA Technologies, Inc. (Coralville, IA) and were PAGE purified. Peracetic acid was obtained from Aldrich as a $32 \%(4.2 \mathrm{M})$ solution in dilute acetic acid. Deuterium- substituted (98\%) tyrosines (ring- $d_{4}$, ring-3,5- $d_{2}$, and $\beta, \beta$ $d_{2}$ ) were from Cambridge Isotope Laboratories (Andover, MA). Biochemicals were from Sigma (St. Louis, MO); NiNTA resin was from Qiagen (Valencia, CA), and BugBuster protein extraction reagent was from Novagen (Madison, WI). The cAOS substrate $8 R$-hydroperoxy- $9 E, 11 Z$-eicosatetraenoic acid $(8 R$-HpETE) was provided by A. R. Brash (Vanderbilt University, Nashville, TN). The nonnatural substrate $13 R$ hydroperoxy-9Z,11E-octadecadienoic acid (13R-HpODE) was provided by E. H. Oliw (Uppsala University, Uppsala, Sweden).

Expression and Purification of Native and Mutant cAOS. The $43 \mathrm{kDa}$ domain of coral AOS, bearing a C-terminal His tag, was overexpressed in Escherichia coli BL21 as described (7) using an expression construct (7) provided by A. R. Brash (Vanderbilt University, Nashville, TN). Site-directed mutagenesis employed sense and antisense primers and was performed using the Stratagene (La Jolla, CA) QuikChange mutagenesis kit protocol. The sense sequences of native cAOS corresponding to the mutagenesis primers, before mutation, are as follows: Y193, 5'GCG CAT CTT TAC TAC TAT TCT CAA GTA ACG ATG CC3'; Y209, 5'GGT AAA GTG CGT TAT TGT AG ATA TCG AGC3'. The Y codons changed are underlined, and these are substituted in mutagenesis primers by CAC for $\mathrm{Y} 193 \mathrm{H}$ and TTC for Y209F. The mutants were confirmed by dideoxy sequencing using a forward primer for the T7 promoter (5'TAA TAC GAC TCA CTA TAG GG3'), which is 116 nucleotides upstream of the Bam $\mathrm{H} 1$ cloning site; a forward primer corresponding to the cAOS nucleotide sequence 472-491 bp downstream of the ATG of the cAOS coding region (5'GAA GGA GAT GCT GCT GAG G3'), and a reverse primer located 85 nucleotides downstream of the $C l a$ I cloning site (5'CCT GAC GTC TAA GAA ACC3').

The published protocol for $\mathrm{cAOS}$ expression was modified (7), in that antibiotic was omitted from the last step of growth in $\mathrm{TB}$ and that cells were lysed using BugBuster protein extraction reagent (Novagen, Madison, WI). Coral AOS mutants were expressed in BL21(DE3) cells, and after the initial culture was diluted into TB, the culture was grown, with rapid shaking, at $37{ }^{\circ} \mathrm{C}$ until it had absorbance at 600 $\mathrm{nm}$ of 1.2. The cells were cooled to $15^{\circ} \mathrm{C}$, induced with $0.05 \mathrm{mM}$ IPTG, and then incubated further at $15^{\circ} \mathrm{C}$ for 24 $\mathrm{h}$, with slow shaking.

Protein Analysis of Native and Mutant cAOS. Aliquots of native and mutant cAOS were purified by reverse-phase HPLC on an analytical C4 column (Phenomenex, $0.46 \times$ $25 \mathrm{~cm}, 0.7 \mathrm{~mL} / \mathrm{min}$ ) using solvents $\mathrm{A}=0.1 \%(\mathrm{v} / \mathrm{v}) \mathrm{TFA}$ in water and $\mathrm{B}=0.07 \%$ TFA in acetonitrile $(5 \mathrm{~min}$ of $25 \%$ solvent B followed by a linear gradient to $75 \%$ solvent B, changing at $0.5 \% / \mathrm{min}$ ). The majority of the samples appeared as one peak that was collected, lyophilized, and dissolved to $50 \mu \mathrm{M}$ in $0.1 \%$ trifluoroacetic acid. The heme signal was absent after RP-HPLC. Sequencing verified that the Nterminal sequences of the native and mutant proteins were ASMTGGQQMG. However, minor sequences were also found in $\mathrm{Y} 193 \mathrm{H}$, corresponding to initiation at methionines downstream of the majority start sequence. The first 15 of the amino acids in the expressed cAOS coding sequence (7) are a leader sequence, containing two other methionines, from the N-terminus of the T7 gene 10 protein (MASMTGGQQMGRGST) (24). The sequence analysis confirms that 
the first methionine is cleaved from the expressed protein. Approximate molecular masses were determined by MALDI/ TOF mass spectral analysis using BSA as an internal calibrant. Repeated measurements of native and mutant cAOS samples, using the internal calibrant, gave the expected molecular masses to within the accuracy of the MALDI/ TOF method $( \pm 0.1-0.2 \%, 44 \mathrm{Da})$. To evaluate deuterium incorporation, masses of native-protonated cAOS and the deuterium-substituted protein (substituted with ring- $d_{4}$ tyrosine) were determined using a $9.4 \mathrm{~T}$ FT-ICR mass spectrometer equipped with an external microelectrospray ionization source $(25,26)$. The $40+$ charge state was the most intense peak, and it was isolated for detailed mass analysis.

Expression of Deuterium-Tyrosine-Substituted cAOS. BL21 cells for native cAOS expression were grown at 37 ${ }^{\circ} \mathrm{C}$ in $\mathrm{LB}$, as reported (7), and were added to 4 volumes of medium containing, in addition to the standard ingredients of TB, deuterium-substituted tyrosine $(0.2 \mathrm{mg} / \mathrm{mL})$ and, to inhibit mixing of isotope into other aromatic amino acids, unsubstituted phenylalanine and tryptophan $(0.05 \mathrm{mg} / \mathrm{mL})$. The supplemented TB culture was grown at $28{ }^{\circ} \mathrm{C}$ for $24 \mathrm{~h}$ and worked up as reported (7).

Optical Measurements of cAOS. Optical measurements were performed on a Hewlett-Packard diode array spectrophotometer (model 8453). cAOS was prepared for examination of the effect of $\mathrm{pH}$ on heme spectra by making a 100fold dilution of $0.58 \mathrm{mM}$ cAOS with the appropriate buffers at $\mathrm{pH} 3-6$ (0.15 $\mathrm{M}$ citrate/phosphate) and at $\mathrm{pH} 7-8(0.1$ $\mathrm{M}$ potassium phosphate). Absorbance measurements were taken $20 \mathrm{~min}$ (room temperature) after the dilution. Conditions of other optical measurements are described in the text.

Oxidation of cAOS. Peracetic acid (4.2 M) was diluted in 4.2 $\mathrm{M}$ acetic acid so that the sum of the concentrations of oxidant and acetic acid, and the $\mathrm{pH}$, in the final protein sample was the same in each sample of a set. Further dilutions of peracetic acid in buffers of $\mathrm{pH}>6.0$ were made immediately before use, and conditions were established so that the final $\mathrm{pH}$ was 6.8-7.0. Typically, for UV/vis spectroscopy, peracetic acid $(24-80 \mathrm{mM}$ in $4.2 \mathrm{M}$ acetic acid) was diluted 100 -fold with $0.2 \mathrm{M}$ potassium phosphate, $\mathrm{pH} 7.0$, and $12.5 \mu \mathrm{L}$ of this solution was added to $987.5 \mu \mathrm{L}$ of cAOS $(3-10 \mu \mathrm{M})$. For typical EPR experiments, $0.32 \mathrm{M}$ peracetic acid in $4.2 \mathrm{M}$ acetic acid was diluted 20 -fold into $0.05 \mathrm{M}$ MES, $\mathrm{pH}$ 6.5, to give $\mathrm{pH}$ 4.0. This solution (5 $\mu \mathrm{L})$ was placed in the bottom of an X-band EPR tube, and 200 $\mu \mathrm{L}$ of cAOS (0.4 mM in 0.1 M MES, pH 7.0) was added forcefully, using a $1.5 \mathrm{~mm}$ OD polyethylene tube attached to a pipet tip. The sample was capped and frozen in liquid nitrogen within 5-15 s, or longer if time was the significant variable.

Early events in the reaction of cAOS with peracetic acid $\left(25^{\circ} \mathrm{C}\right)$ were monitored by time-resolved changes in cAOS absorbance at 406 (native), 660 (compound I), and $435 \mathrm{~nm}$ (intermediate II), using a KinTek rapid-mix, stopped-flow instrument. Five hundred points were collected in windows of either 0.1 or $2.0 \mathrm{~s}$.

Reactions of cAOS with hydrogen peroxide or $m$-chloroperbenzoic acid were also monitored by absorbance changes at $406 \mathrm{~nm}$. Solutions of $m$-chloroperbenzoic acid were made up in $50 \%$ absolute ethanol immediately prior to use. A solution $(10 \mu \mathrm{L})$ of $m$-chloroperbenzoic acid was added in 1-, 5-, 20-, and 40-fold excess to $990 \mu \mathrm{L}$ of 0.22 $\mathrm{mM}$ cAOS in 0.1 M MES, pH 7.

Enzymatic Activity of cAOS. Rates of $8 R$-HpETE $(\epsilon 235$ $\mathrm{nm}, 25000)$ turnover at $\mathrm{pH} 7.9(50 \mathrm{mM}$ Tris- $\mathrm{HCl})$ were determined with $2.5-2.7 \mathrm{nM}$ native and mutant cAOS. Cuvette path lengths for different substrate concentrations were $10 \mathrm{~mm}(7-25 \mu \mathrm{M}), 5 \mathrm{~mm}(45-80 \mu \mathrm{M})$, and $2 \mathrm{~mm}$ $(100-150 \mu \mathrm{M})$. Reactions were initiated by rapid addition of 100 volumes of substrate to 1 volume of enzyme. Initial rates were obtained by linear fits to data points in the first $2-12 \mathrm{~s}$ after mixing. Inactivation of native and mutant cAOS, after a limited number of turnovers, was also examined using the nonnatural substrate, $13 R$-HpODE. cAOS $(40-80 \mathrm{nM})$ and $13 R$-HpODE $(63-90 \mu \mathrm{M})$ were mixed in Tris- $\mathrm{HCl}$ buffer (50 mM, pH 7.9) in differing proportions. The HpODE remaining was determined from the absorbance at $235 \mathrm{~nm}$ $\left(\epsilon 25000 \mathrm{M}^{-1} \mathrm{~cm}^{-1}\right)$, after a $2 \mathrm{~h}$ incubation period at room temperature, by which time substrate turnover had ceased.

EPR Spectroscopy. EPR spectra at X-band $(\sim 9.28 \mathrm{GHz})$ were recorded on a Varian E 109 spectrometer equipped with an Oxford Instruments ESR9/10 pumped, gas-flow cryostat. Typical conditions for recording radical signals were $10 \mathrm{mT}$ scan range, $80 \mathrm{~K}, 0.16 \mathrm{mT}$ modulation amplitude, and $1 \mathrm{~mW}$ power. Those for recording the heme signal at $80 \mathrm{~K}$ were $400 \mathrm{mT}$ scan range, $0.16 \mathrm{mT}$ modulation amplitude, and 1 $\mathrm{mW}$ power. The figure legends contain other relevant instrumental parameters. X-Band frequencies were measured at the bridge. Values of the $g$-factor at the sample were calibrated using the spectrum obtained by freezing a sealed tube $(0.5 \mathrm{~mm}$ i.d.) of $\mathrm{CaO}$ containing a manganous impurity (standard from Bruker BioSpin) in 0.2 M aqueous buffer (MES, pH 7.0) in a $4 \mathrm{~mm} \mathrm{X-band} \mathrm{quartz} \mathrm{EPR} \mathrm{tube.} \mathrm{Values}$ of buffer $\mathrm{pH}$ cited for EPR samples were measured at room temperature $\left(\sim 22{ }^{\circ} \mathrm{C}\right)$.

Simulations of EPR Spectra. Line shape simulations were performed using the XSophe program developed by G. R. Hanson and co-workers (available from Bruker BioSpin). Some calculations of tyrosine radical spectra were shortened by including only one $\beta$-proton (or deuteron) and two ring3,5 protons (or deuterons). When EPR spectra of deuterated samples were simulated, the proton hyperfine couplings were reduced by the ratio of the deuteron/proton magnetic moments $\left(\mu_{\mathrm{D}} / \mu_{\mathrm{H}}=0.1535\right)$. Values of the simulation parameters for tyrosine radical spectra are given in Table 1. Line widths are not adjusted for the presence of deuterium. Calculations on an SGI $\mathrm{O}_{2}$ using the XSophe program, with matrix diagonalization and a Sophe grid of 20 spatial segments and 64 field partitions, required about $15 \mathrm{~min}$ for splitting by four protons $(I=1 / 2)$ and about $2.5 \mathrm{~h}$ for two protons and two deuterons $(I=1)$.

\section{RESULTS}

Stability of cAOS with Variation in $\mathrm{pH}$ and Oxidant. Native cAOS exhibits UV/vis maxima at wavelengths of 280, 406, 500,540 , and $620 \mathrm{~nm}$, and the ratio of absorbances $406 \mathrm{~nm} /$ $280 \mathrm{~nm}$ is $\sim 1.5(8)$. The ionization of peracetic acid affects the rate of reaction with other hemoproteins $(27,28)$, the rate being faster at $\mathrm{pH}$ values where the oxidant is not ionized, so the $\mathrm{pH}$ stability of cAOS was examined to establish conditions for reactions with oxidant. The optical spectra of cAOS are essentially unchanged in the $\mathrm{pH}$ range 
Table 1: Comparison of Simulation Parameters for Tyrosine Radical EPR Spectra of $\mathrm{CAOS}^{a}$ and $\mathrm{BLC}^{b}$

\begin{tabular}{lll}
\hline & \multicolumn{1}{c}{$\mathrm{cAOS}^{a}$} & \multicolumn{1}{c}{$\mathrm{BLC}^{b}$} \\
\hline$g_{x}$ & 2.00750 & 2.00777 \\
$g_{y}$ & 2.00450 & 2.00460 \\
$g_{z}$ & 2.00232 & 2.00232 \\
$\beta_{1} A_{x}, A_{y}, A_{z}(\mathrm{MHz})$ & $48,44,44$ & $20,15.5,16$ \\
$\beta_{2} A_{x}, A_{y}, A_{z}(\mathrm{MHz})$ & near $0^{c}$ & $14.5,11.9,10.6$ \\
$\theta_{1}, \theta_{2}(\mathrm{deg})^{d}$ & +21 to $30,-99$ to -90 & $+57,-63$ \\
ring-3,5 $A_{x}, A_{y}, A_{z}(\mathrm{MHz})$ & $-26.5,-7.2,-19$ & $-25.9,-7.2,-19.5$ \\
$\Phi_{3,5^{d}}(\mathrm{deg})$ & \pm 25 & \pm 23 \\
ring-2,6 $A_{x}, A_{y}, A_{z}(\mathrm{MHz})$ & $6,7.5,5^{c}$ & $5,7.4,<3$ \\
$\Phi_{2,6^{d}}(\mathrm{deg})$ & \pm 10 & \pm 10
\end{tabular}

${ }^{a}$ This work. The microwave frequency in simulations is $9.28 \mathrm{GHz}$. The components of the anisotropic line widths are (Gaussian $w_{x}, w_{y}$, $\left.w_{z}\right) 0.40,0.25$, and $0.28 \mathrm{mT} .{ }^{b}$ From ref $31 .{ }^{c}$ Calculated; see text. ${ }^{d}$ The $g_{z}$ and aromatic proton $A_{z}$ axes are perpendicular to the aromatic ring. The dihedral angle $\theta_{1}$ between the $\beta$-proton $\mathrm{C}-\mathrm{H}$ bonds and the normal to the ring plane was estimated by taking spin densities on $\mathrm{C}_{1}$ of $0.32-$ 0.37 (see text). Values of $\theta_{2}$ reported are $\theta_{1}-120^{\circ}$. Angles $\Phi_{3,5}$ and $\Phi_{2,6}$ are the angles between the directions $A_{y}$ for the aromatic proton hyperfine and the electron $g_{y}$. The $g_{x}$ direction is parallel the ring $\mathrm{C}_{4}-\mathrm{O}$ bond.

$8-6$, and at $\mathrm{pH} 5$, the heme absorbance at $406 \mathrm{~nm}$ is $\sim 13 \%$ decreased. At $\mathrm{pH} 3$ and 4, some precipitation occurs gradually, and the heme absorbance of the sample, clarified by centrifugation, is irreversibly replaced at these low $\mathrm{pH}$ values by a broad absorbance centered at $\sim 370 \mathrm{~nm}$. The cAOS high-spin, ferric heme EPR signal, shown for $\mathrm{pH} 8.0$ in ref 8 , was also examined in samples with a range of $\mathrm{pH}$ values. The low-field $g_{y^{-}}$and $g_{x}$-values are separated increasingly, or the rhombicity $(E / D)$ is increased, as $\mathrm{pH}$ is lowered to 5.5. This increase in rhombicity of the iron environment at low $\mathrm{pH}$ also occurs in BLC (29), and it is less pronounced in cAOS. The values of $E / D$, determined by simulation of EPR spectra, for cAOS are 0.0295 at $\mathrm{pH} 5.5(0.25 \mathrm{M}$ sodium acetate) and 0.027 at $\mathrm{pH} 7.9(0.25 \mathrm{M}$ Tris- $\mathrm{HCl})$. The values of $E / D$ determined for BLC are 0.0355 at $\mathrm{pH} 5.5$ and 0.0235 at $\mathrm{pH} 7.9$ (same buffers).

The UV/vis absorbance of cAOS $(4-8 \mu \mathrm{M}, \mathrm{pH} 7.0)$ appeared unchanged, after $30 \mathrm{~s}$, upon addition of 1 molar equiv of peracetic acid (adjusted to $\mathrm{pH}$ 7.0), but absorbance at $406 \mathrm{~nm}$ declined with additions of higher levels of oxidant, and a 10 min incubation period, as illustrated in Figure 1. The changes after $10 \mathrm{~min}$ are irreversible. The optical spectra of $4.2 \mu \mathrm{M}$ cAOS, treated with 1 molar equiv of oxidant, also remain unchanged after 2 days at $4{ }^{\circ} \mathrm{C}$. The Figure 1 inset shows optical spectra of untreated cAOS (dashed line) and cAOS 10 min after treatment with 10 molar equiv of peracetic acid (solid line). Besides decreased absorbance at $406 \mathrm{~nm}$, the absorbance maxima at 500 and $620 \mathrm{~nm}$ also decrease with higher than 1 equiv of peracetic acid added. The absorbance at $280 \mathrm{~nm}$ appears shifted upward because of the absorbance of the acetate added.

Optical Detection of Oxidized Heme Intermediates. Timedependent changes in cAOS absorbance when 10 molar equiv of peracetic acid is added were monitored by rapid-mix, stopped-flow at $\mathrm{pH} 7.0(0.1 \mathrm{M}$ sodium phosphate $)$ and 25 ${ }^{\circ} \mathrm{C}$. The absorbance at $406 \mathrm{~nm}$ underwent a rapid initial exponential decrease at $\sim 350 \mathrm{~s}^{-1}$ with a partial recovery after $0.8-1.5 \mathrm{~s}$. Time-dependent absorbance changes were also measured at wavelengths characteristic of catalase compound I (660 nm) and intermediate II (435 nm) (22). Initially, absorbance at $660 \mathrm{~nm}$ increased at the rate that $406 \mathrm{~nm}$

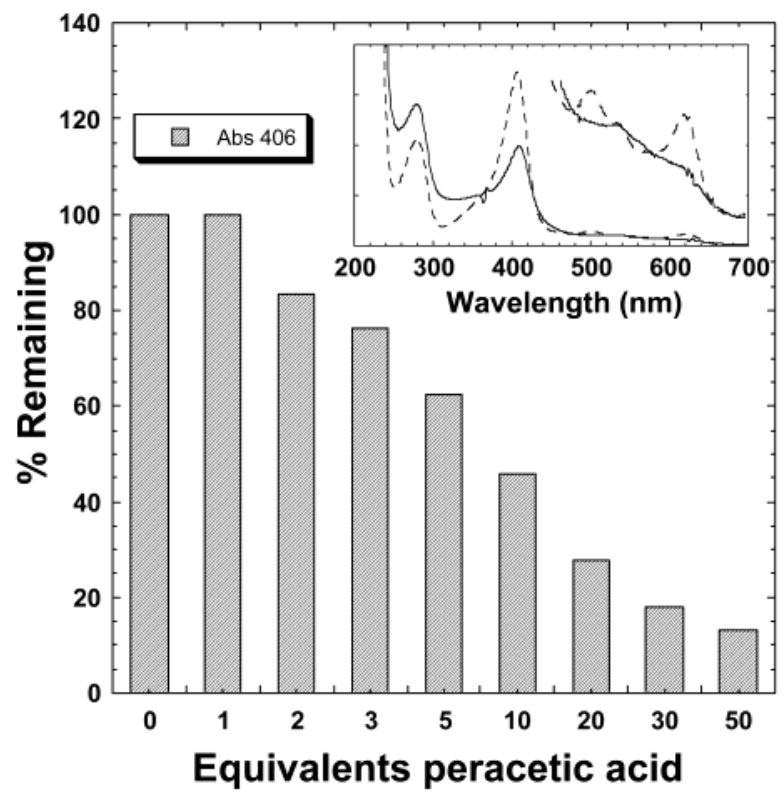

FIGURE 1: Optical spectra of cAOS treated with peracetic acid. The bar graph gives the absorbance at $406 \mathrm{~nm}$ of cAOS $(4.2 \mu \mathrm{M}$ in 0.2 M MES, $\mathrm{pH} 7.0) 10 \mathrm{~min}$ after addition of the indicated peracetic acid molar equivalents. Peracetic acid was added to fresh, untreated cAOS samples for each data bar. The spectra of two of the samples are shown in the inset: untreated cAOS (dashed line) and cAOS treated with 10 molar equiv of peracetic acid and stored for $18 \mathrm{~h}$ (solid line). The long-wavelength region is amplified $10 \times$ compared to the full spectrum.

absorbance decreased $\left(350 \mathrm{~s}^{-1}\right)$. The $660 \mathrm{~nm}$ absorbance decreased slowly until about $0.7 \mathrm{~s}$ and then decreased more rapidly as the absorbance at $406 \mathrm{~nm}$ recovered. Monitoring $435 \mathrm{~nm}$ absorbance revealed an intermediate with absorbance that increased at $\sim 13 \mathrm{~s}^{-1}$ and then decreased at $\sim 3 \mathrm{~s}^{-1}$. Representative absorbance changes at these three wavelengths, over a $2 \mathrm{~s}$ period, are illustrated in Figure 2. The faster changes were monitored in a window of $0.1 \mathrm{~s}$ (data not shown).

Reactions, at $\mathrm{pH} 7.0$, of cAOS with $m$-chloroperbenzoic acid and with hydrogen peroxide were also monitored via cAOS absorbance at $406 \mathrm{~nm}$. After a 10 min incubation period, the $406 \mathrm{~nm}$ absorbance was unchanged by addition of 1 equiv of $m$-chloroperbenzoic acid; however, addition of 20 molar equiv or more of this oxidant results in loss of absorbance at $406 \mathrm{~nm}$ and in a transient peak appearing at $720-735 \mathrm{~nm}$. The latter may be similar to a transient peak observed in oxidation of horseradish peroxidase by $\mathrm{m}$ chloroperbenzoic acid, but in that case absorbances attributed to compound I and verdoheme are observed at 965 and 670 $\mathrm{nm}$ (30). Reaction of cAOS with a 10-fold molar excess of hydrogen peroxide also results in loss of about half the 406 $\mathrm{nm}$ optical absorbance, but the time required is $1.5 \mathrm{~h}$.

Formation of a Tyrosine Radical in Oxidized cAOS. The effects of cAOS oxidation by peracetic acid (1-50 molar equiv, final $\mathrm{pH}$ 6.8-7.0), followed by rapid freezing $(<10$ s), were examined further by EPR spectroscopy. The amplitude of the first-formed radical EPR signal does not increase on addition of more than 1 equiv of peracetic acid; however, the amount of recovered high-spin ferric heme signal decreases as the excess of oxidant increases. The time window for trapping a radical with EPR hyperfine splitting is about $15 \mathrm{~s}$ at $4{ }^{\circ} \mathrm{C}$. However, by $30 \mathrm{~s}$ after mixing, the 


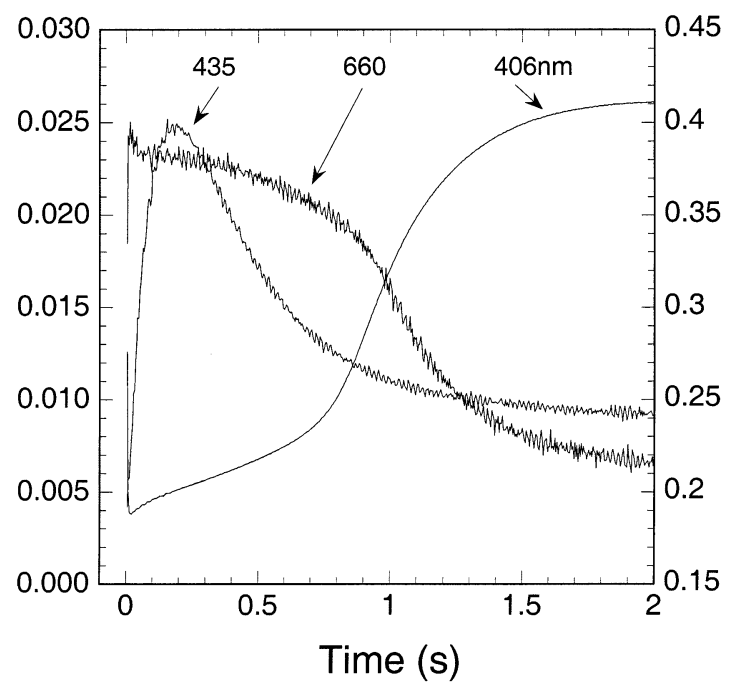

FIGURE 2: Immediate time-dependent changes in absorbance following oxidation of cAOS. Ten volumes of cAOS $(11.6 \mu \mathrm{M}$ in $0.1 \mathrm{M} \mathrm{P}, \mathrm{pH} 7.0)$ and 1 volume of peracetic acid (1.16 mM in 0.1 $\mathrm{M} \mathrm{P}_{\mathrm{i}}, \mathrm{pH}$ 7.0) were mixed by stopped flow. Changes in absorbance were monitored at $4 \mu$ s intervals at $406 \mathrm{~nm}$ (native ferric heme), $660 \mathrm{~nm}$ (compound I), and $435 \mathrm{~nm}$ (intermediate II). Initial values of the absorbances at these wavelengths were $0.58(406 \mathrm{~nm}), 0.092$ (435 $\mathrm{nm})$, and $0.001(660 \mathrm{~nm})$. Due to rapid changes within $4 \mu \mathrm{s}$ after mixing, the traces in the figure do not reflect the initial values. About $80 \%$ of the initial $406 \mathrm{~nm}$ absorbance is recovered at $2 \mathrm{~s}$, and then further irreversible change occurs to the values indicated in Figure 1. The left $y$-axis scale applies to absorbance at 435 and $660 \mathrm{~nm}$. The right $y$-axis scale applies to $406 \mathrm{~nm}$ absorbance.

EPR signal with hyperfine splitting has begun to decay, and samples frozen $1 \mathrm{~min}$ after addition of oxidant exhibit a second radical signal of $1.0 \mathrm{mT}$ peak-to-peak width with no hyperfine splitting, also centered at about $g=2.004-5$. When excess oxidant is used, progression to the featureless radical signal is faster (Figure 3). Samples held at $4{ }^{\circ} \mathrm{C}$ for longer than $\sim 90 \mathrm{~s}$, after oxidation by 1 equiv, have highspin ferric heme signals but no radical signals. Together, the EPR and the optical measurements show that the amount of ferric cAOS has already returned to a final value shortly after peracetic acid is added, and transient radicals remain for a longer period. With greater than 1 molar equiv of oxidant, the amplitude of the recovered ferric heme EPR signal diminishes (not shown), but the radical signals remain as shown in Figure 3. The diminution of the high-spin ferric EPR signal parallels the reduction of the optical absorbance at $406 \mathrm{~nm}$ (Figure 1).

EPR of cAOS Containing Deuterated Tyrosine. The major radical in oxidized cAOS is identified as tyrosyl on the basis of changes in EPR spectra when deuterium-substituted tyrosine is incorporated in the expressed protein (Figure 4). The experimental spectra (Figure $4 \mathrm{~A}-\mathrm{C}$, upper spectra) are compared with computer simulations (Figure $4 \mathrm{~A}-\mathrm{C}$, lower spectra) to assign characteristic EPR parameters. The EPR spectrum of unsubstituted cAOS is fit by hyperfine splittings and $g$-values within the range known for tyrosine radicals in other proteins and models $(31-34)$. Most of the calculations included only the three protons of largest hyperfine splitting (the $\beta_{1}$ and the 3,5-ring protons), but a few simulations that included all six protons of the spin system were performed, and they differed little from simplified simulations.

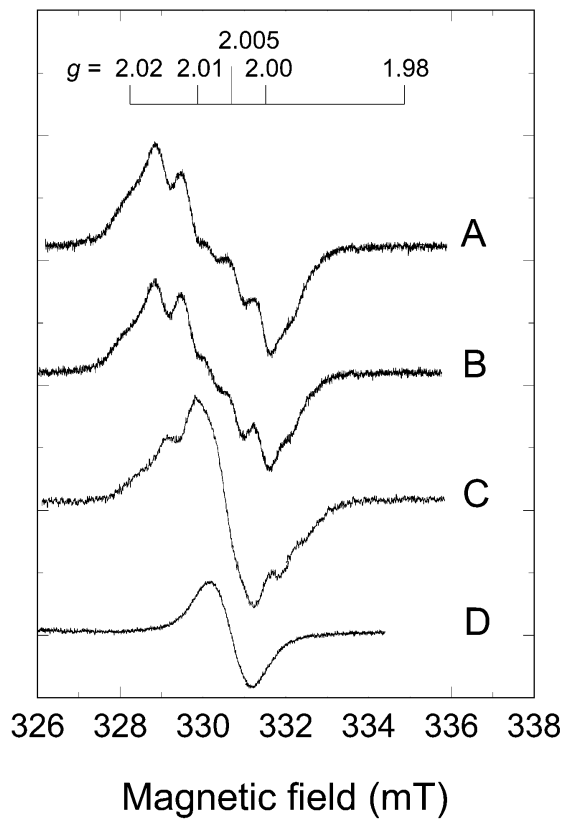

FIGURE 3: EPR spectra $(9.28 \mathrm{GHz})$ showing results of increasing equivalents of oxidant added to cAOS. Samples were prepared at $4{ }^{\circ} \mathrm{C}$ and trapped by freezing in the EPR tube $<10 \mathrm{~s}$ after mixing. Spectra were recorded at $80 \mathrm{~K}$. Molar ratios of peracetic acid to cAOS (0.24-0.25 mM) were (A) 5:1, (B) 10:1, (C) 30:1, and (D) 50:1. Instrument settings common to all spectra are as follows: temperature, $80 \mathrm{~K}$; modulation amplitude, $0.16 \mathrm{mT}$; microwave power, $1 \mathrm{~mW}$; gain, 20000; time constant, $0.25 \mathrm{~s}$; scan rate, 20 $\mathrm{mT} / \mathrm{h}$.

High-resolution mass data were obtained by FT-ICR mass spectrometry for native and deuterium-substituted cAOS. The isolated $40+$ peaks had maxima corresponding to $\sim 44680$ (mass of 40 protons removed) for native cAOS and $\sim 44720$ for a sample with ring- $d_{4}$ tyrosine incorporated. The isolated $40+$ peak of the deuterium-substituted sample was about 4 times wider than from the unsubstituted sample. These results are consistent with slightly over 50\% incorporation of deuterated tyrosine, distributed randomly over the 18 tyrosines in the cAOS sequence.

cAOS Tyrosine Mutations. The location of the radical formed in cAOS was examined by site-directed mutagenesis and oxidation. Relevant sequences around tyrosines common to $\mathrm{AOOS}$ and BLC guided the choice of mutation sites, and these are compared in Table 2, together with the analogous sequences in P. mirabilis catalase (PMC) (22). PMC lacks the tyrosines corresponding to cAOS Y193 and Y209, but mutants, in which Tyr replaces the native Phe at sites in PMC corresponding to cAOS Y193 and Y212, have been reported, and these mutant PMC sequences are also indicated in Table 2. [Mutations at cAOS Y269, in a second sequence with similarity to the BLC sequence, were not prepared because the corresponding site in BLC (Y324) is not a proposed radical site.] The purity of native and mutant cAOS, after Ni-NTA resin and anion-exchange chromatography steps, was monitored by reverse-phase (RP) HPLC (Table 3) and was $>87 \%$.

The molecular masses of RP-HPLC-purified cAOS and mutants were determined by MALDI/TOF mass spectrometry, and the N-terminal sequences were determined by amino acid sequencing. Both analyses were consistent with expected masses of native and mutant cAOS with a 14 amino 


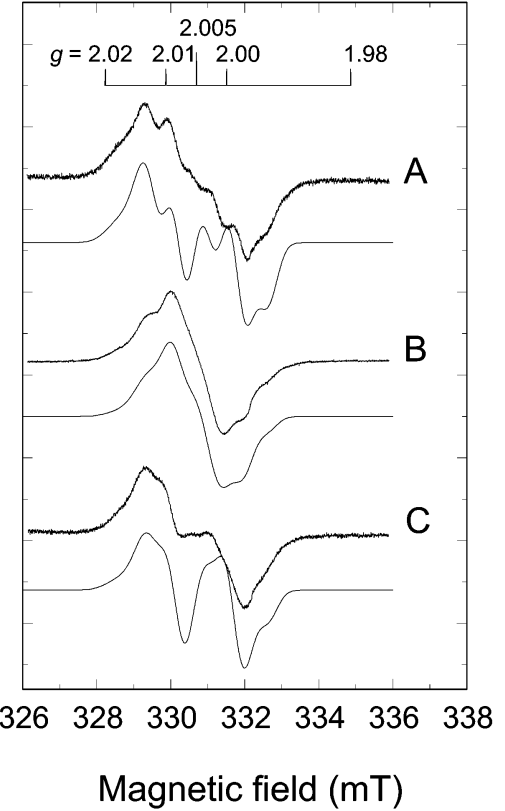

FIGURE 4: Experimental and calculated EPR signals $(9.28 \mathrm{GHz})$ of the radical formed on peracetic acid treatment of $\left[{ }^{1} \mathrm{H}\right]-$ and $\left[{ }^{2} \mathrm{H}\right]-$ Tyr-substituted cAOS. In each pair of spectra, the upper one is the experimental and the lower is the calculated spectrum. In the experimental samples, 1 equiv of peracetic acid is added to cAOS and the final $\mathrm{pH}$ is $6.8-7.0$. (A) The radical formed in fully protonated cAOS $(0.42 \mathrm{mM}$; instrument gain, 25000). (B) The radical formed in cAOS partially substituted with deuterium in the tyrosine $\beta, \beta$-protons $(0.85 \mathrm{mM}$; gain 3200$)$. (C) The radical formed in cAOS partially substituted with ring- $d_{4}$ tyrosine $(0.27 \mathrm{mM}$; gain 20000). Other instrument settings are those given in the Figure 3 legend. Parameters for the simulations are given in Table 1. The calculated spectra are the sums of a deuterium-substituted tyrosine radical and a fully protonated radical $[80 \%$ D-substituted in (B) and 50\% D-substituted in (C)]. The line shape in (B) cannot clearly be distinguished from the background signals, shown earlier in Figure 3C,D.

Table 2: Sequences of cAOS and Catalases in Which Tyrosine Radical Formation ${ }^{a}$ Was Examined

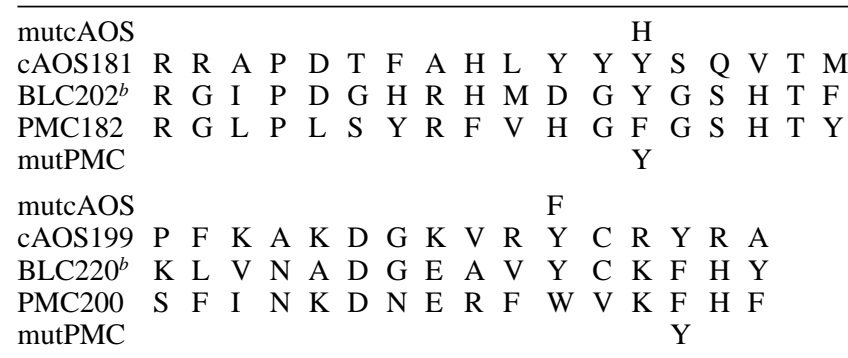

${ }^{a}$ Sites of radical formation in BLC discussed in refs 20-23; PMC mutations and radical formation are described in ref $22 .{ }^{b}$ The reported sequence of BLC was determined by Edman amino acid analysis (45). BLC amino acids 212 and 225 were found to be D by this method, as listed above. However, the human liver catalase sequence (AAK29181), translated from the nucleotide sequence, has $\mathrm{N}$ in both of these positions. The occurrence of $\mathrm{G}$ immediately after each $\mathrm{N}$ makes deamidation at 212 and 225 a likely posttranslational modification of BLC (46). Thus the nucleotide and protein sequences may differ in this regard.

acid $\mathrm{N}$-terminal leader and the $\mathrm{C}$-terminal $\mathrm{His}_{4}$ tag appended (Table 3).

Initial measurements of the kinetic constants for native and mutant cAOS reactions with $8 R$-HpETE $(\mathrm{pH} 7.9$ and $21{ }^{\circ} \mathrm{C}$ ) showed that there was no more than a factor of 2
Table 3: Characterization of Native cAOS and Mutants

\begin{tabular}{lcccc}
\hline & & \multicolumn{2}{c}{ MW } & turnovers to $^{\text {turnactivate }}$ \\
\cline { 3 - 4 } & Abs ratio $^{a}$ & expected $^{b}$ & found $^{c}$ & inactivan $^{2}$ \\
\hline native cAOS & 1.5 & 44679 & $446(81)$ & $1071 \pm 70$ \\
Y209F & $1.3-1.4$ & 44663 & $446(62)$ & $820 \pm 72$ \\
Y193H & $1.3-1.4$ & 44637 & $446(36)$ & $546 \pm 29$ \\
\hline
\end{tabular}

${ }^{a}$ Absorbance ratio: $406 \mathrm{~nm} / 280 \mathrm{~nm} .{ }^{b}$ Calculated for amino acids $1-373$ of the coral fusion protein, with 14 other amino acids appended $\mathrm{N}$-terminally and 4 His appended C-terminally (see text) (7). ${ }^{c}$ Mass spectral analyses by MALDI/TOF with an internal calibrant are on the order of $\pm 44 \mathrm{Da} .{ }^{d}$ In reaction with the nonnatural substrate $13 R$ HpODE. Average \pm SE $(n=3)$. See Figure $5 c$ legend for experimental conditions.

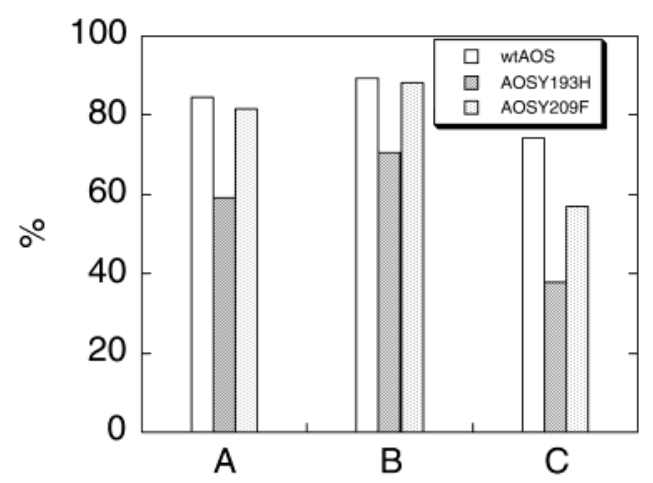

FIGURE 5: The percent of $13 R$-HpODE consumed (ordinate) after a $2 \mathrm{~h}$ incubation, at room temperature, with coral AOS is shown for different initial concentrations (abscissa). The initial concentrations of hydroperoxide and cAOS are (A) $90 \mu \mathrm{M} H \mathrm{HpODE}$ and 77 $\mathrm{nM}$ cAOS, (B) $63.4 \mu \mathrm{M}$ HpODE and $77 \mathrm{nM}$ cAOS, and (C) 63.4 $\mu \mathrm{M}$ HpODE and $44 \mathrm{nM}$ cAOS. Samples were in Tris $(0.05 \mathrm{M}, \mathrm{pH}$ 7.9). Key: open bars (wt cAOS), dark shaded bars (Y193H cAOS), and lightly shaded bars (Y209F cAOS).

difference in $K_{\mathrm{m}}$ and $V_{\max }$, between mutant and native cAOS. Although $8 R$-HpETE is the natural substrate of coral AOS, the enzyme is able to turn over other unsaturated fatty acid hydroperoxides. The number of turnovers of $13 R-H p O D E$, before inactivation, was measured to further characterize the enzymatic activity of coral AOS and mutants. The percentage of hydroperoxide consumed after turnover had ceased $(2 \mathrm{~h}$ ) was determined for several concentrations of enzyme and substrate (Figure 5). In all cases, the Y193H cAOS mutant turned over fewer molecules of $13 R$-HpODE before it inactivated.

EPR spectra of the samples formed by peracetic acid oxidation of native cAOS and the mutant protein, cAOS Y193H, are compared in Figure 6. For Figure 6, the spectra were recorded at $3.5 \mathrm{~K}$ to amplify the signals of the dilute samples $(0.09 \mathrm{mM})$. Note, by comparison with spectra in Figures 3 and 4, that the EPR signal of oxidized native cAOS has a broader line width at $3.5 \mathrm{~K}$ than at $80 \mathrm{~K}$. Almost no radical signal is observed in oxidized $\mathrm{Y} 193 \mathrm{H}$. EPR spectra of $0.4 \mathrm{mM}$ native and $\mathrm{Y} 193 \mathrm{H}$ mutated cAOS were also compared at $80 \mathrm{~K}$. The native protein spectrum is the same as in previous measurements at $80 \mathrm{~K}$ (Figure 4A), but only a small, narrow signal was observed from the mutant (not shown). The EPR spectrum of the oxidized Y193H sample does retain a high-spin ferric heme signal, however (not shown). Additionally, oxidized native cAOS and Y209F have identical radical signals (not shown). The Y193H mutant was examined twice in a series of experiments in which EPR spectra of native cAOS, Y193H, and native cAOS were 


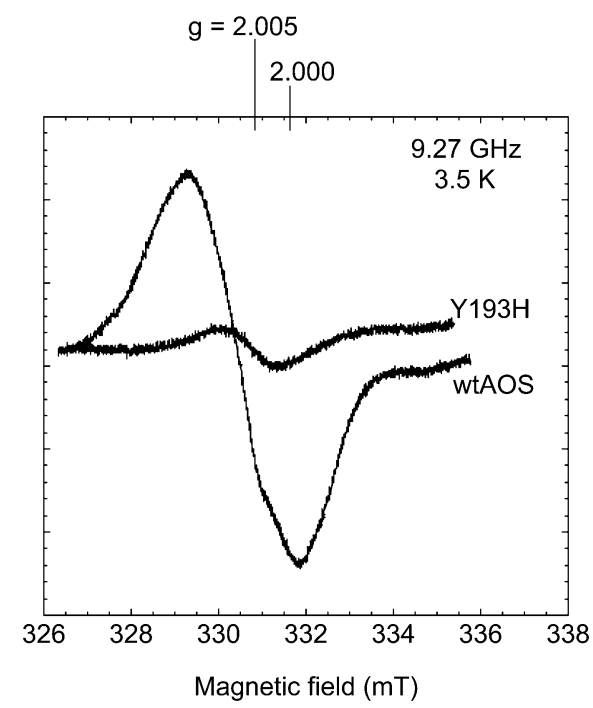

FIGURE 6: Low-temperature EPR spectra comparing oxidized native cAOS and $\mathrm{Y} 193 \mathrm{H}$ mutant. Both protein samples were $0.09 \mathrm{mM}$ and were oxidized with 2 molar equiv of peracetic acid. Instrument conditions are as follows: temperature, $4 \mathrm{~K}$; modulation amplitude, $0.16 \mathrm{mT}$; gain, 20000; microwave power, $1 \mathrm{~mW}$; time constant, $0.25 \mathrm{~s}$; scan rate, $20 \mathrm{mT} / \mathrm{h}$.

recorded sequentially to rule out possible problems arising from sample preparation or instrumentation.

\section{DISCUSSION}

Stoichiometric Oxidation of Coral AOS by Peracetic Acid. Coral AOS reacts very rapidly with the hydroperoxide substrate $8 R$-HpETE and about 10-100 times more slowly with other regioisomers of HpETE (7). During these reactions, cAOS inactivates irreversibly after tens of seconds (7). Although the proposed mechanism of allene oxide synthases involves homolytic cleavage of hydroperoxide, many hemoproteins react with organic hydroperoxides by a mixture of homolysis and heterolysis (18). Thus, heterolysis, leading to inappropriate heme intermediates for the cAOS reaction, is a possible mode of inactivation of the enzyme. We reported that a radical is formed when peracetic acid reacts with cAOS (8), and this observation could indicate that a heme compound I intermediate had converted to an intermediate II, in which an amino acid side chain had reduced the porphyrin cation radical in compound I. Examination, by stopped-flow mixing, of cAOS reacting with excess peracetic acid reveals that the reaction indeed passes through compound I, intermediate II, and partially recovered ferric heme in about $1 \mathrm{~s}$ (Figure 2). The radical persists for $>15 \mathrm{~s}$. Reaction of cAOS with a 10-fold molar excess of hydrogen peroxide, at $\mathrm{pH}$ 7.0, also results in loss of about half of the heme absorbance, but the reaction is very slow $(1.5 \mathrm{~h})$, and $m$-chloroperbenzoic acid can serve as an oxidant as well. Thus a variety of peroxy compounds, from fatty acid hydroperoxides to small oxidants, can interact with the cAOS heme in reactions of vastly different rates. These reactions are reminiscent of reactions of catalases with peracetic acid. After peracetic acid oxidation of BLC, compound I decays in less than $15 \mathrm{~s}$ to an intermediate II (20). The lifetime of compound I of some other catalases, for instance, Micrococcus luteus and $P$. mirabilis catalases, is longer $(19,22)$.

Assignment of the Parameters of the cAOS Radical EPR Signal. The evidence obtained that, initially, one tyrosine is selectively oxidized in cAOS includes (a) satisfactory simulations of EPR spectra of the cAOS radical from both protonated and deuterated protein, (b) the near absence of a radical EPR signal upon oxidation of $\mathrm{Y} 193 \mathrm{H}$, and (c) a radical EPR signal of the same shape and intensity, freezetrapped $10-15 \mathrm{~s}$ after addition of either 1 or 10 molar equiv of peracetic acid.

(a) Simulation of cAOS Radical EPR Signals. Characteristic EPR parameters of the major cAOS radical are within the range found for tyrosine radicals in other proteins, including, in catalases, $\mathrm{Y}_{\mathrm{D}}$ and $\mathrm{Y}_{\mathrm{Z}}$ of photosystem II, and ribonucleotide reductases of different origins (summarized in Table 1 of ref 31), and in prostaglandin synthases (PGHS-1 and PGHS-2) $(33,34)$. Most striking is the wide cAOS spectrum (Figure 3) compared to the narrower signals observed in oxidized catalases $(20,22)$. In breadth, the cAOS radical spectrum is similar to the wide doublet EPR radical signal seen in activated PGHS (33). An estimate of EPR parameters characteristic of the cAOS radical (Table 1) is based on simulation of the radical spectra of selectively deuterium-substituted cAOS and of the fully protonated protein. Six proton hyperfine splittings contribute to the EPR spectra of tyrosine radicals, although, in the cAOS radical, the major contributions arise from only three, one $\beta$-methylene proton and the two aromatic ring-3,5 protons. The contribution of the other $\beta$-methylene proton is constrained by bonding geometry and theory to be small. The magnitude of proton hyperfine splittings in EPR signals of delocalized aromatic radicals is related to the unpaired electron spin density on the carbon to which the proton is attached (ring protons) or on the aromatic carbon to which $\mathrm{C}-\mathrm{H}$ is attached ( $\beta$-protons) $(35,36)$. The angle between the two $\mathrm{C}_{\beta}-\mathrm{H}$ bonds is $120^{\circ}$, and the angle they make with the aromatic ring $\pi$-orbital axis (heme normal) is designated $\theta_{1}$ or $\theta_{2}$. The relationship between the $\beta$-proton isotropic hyperfine splitting, $A_{\text {iso }}$, the spin density on aromatic $\mathrm{C}_{1}, \rho_{\pi}$, and the relevant $\theta$ is given by a relation (36) which simplifies to

$$
A_{\text {iso }} \approx \rho_{\pi}\left(B_{2} \cos ^{2} \theta\right)
$$

In eq 1, the value of $B_{2}$ is $\sim 162 \mathrm{MHz}$ (36). The value of $A_{\text {iso }}$ for the larger $\beta$-proton splitting in the cAOS radical spectrum is $45.33 \mathrm{MHz}$, and $\theta_{1}$ refers to the orientation of the proton making this contribution. For $A_{\text {iso }}$ of this magnitude in a tyrosine radical, $\theta_{1}$ is considerably smaller than $60^{\circ}$. As a result, $\theta_{2}$ is close to $90^{\circ}\left(\cos ^{2} \theta_{2}\right.$ is close to zero) so two, independent equations (eq 1) cannot be obtained, and $\rho_{\pi}$ and $\theta_{2}$ cannot be determined independently. An initial estimate of $\theta_{1}$ can be made by employing spin densities, $0.32-0.37$, established at $C_{1}$ of other tyrosine radicals (37). On this basis, the estimated $\theta_{1}$ for the tyrosine radical in cAOS is 21 to $30^{\circ}$ and, therefore, the $\theta_{2}$ range is $-99^{\circ}$ to $-90^{\circ}$, respectively. In contrast, EPR-based estimates of $\theta_{1}$ and $\theta_{2}$ for the BLC tyrosine radical are $57^{\circ}$ and $-63^{\circ}(31)$. The adjacent $\mathrm{C}_{\beta}-\mathrm{C}_{\gamma}$ bond rotamer influences the conformation of the $\mathrm{C}_{1}-\mathrm{C}_{\beta}$ bond, due to steric interaction between the bulky aromatic ring and the amide nitrogen or carbonyl carbon (38). The value of $\theta_{1}$ found for the cAOS tyrosine radical is most consistent with the gauche- $\mathrm{C}$ anti-N rotamer about the $\mathrm{C}_{\beta}-\mathrm{C}_{\gamma}$ bond in a free tyrosine radical. A number of other steric interactions will, of course, also contribute to 
the preferred conformation of the cAOS protein-bound tyrosine.

The hyperfine splittings of the ring-2,6 protons of the cAOS radical are too small to determine accurately by simulation of the cw EPR spectra. The hyperfine splitting values in Table 1 for the ring-2,6 protons have been constrained to satisfy an empirical relation for ring hyperfine splittings in phenoxy radicals, $\mid A_{\text {iso }}($ ring- 3,5$)+A_{\text {iso }}($ ring- 2,6$) \mid$ $=13.2 \pm 0.6 \mathrm{MHz}$ (36).

Deuterium-labeled tyrosine was incorporated in cAOS by adding excess labeled tyrosine at the last transfer of the culture to new medium (a 1:5 dilution), so that less than $100 \%$ labeling is expected. Qualitatively, the EPR spectra of the radicals in deuterium-substituted cAOS (Figure 4B,C) show marked changes for each substitution. FT-ICR mass measurements of ring- $d_{4}$ tyrosine-substituted cAOS indicated about 50\% isotope incorporation. The fits to experimental tyrosine radical spectra in Figure $4 \mathrm{C}$ were made by adding calculations for $50 \%$ deuterium in the ring- $d_{4}$ protons and for $50 \%$ fully protonated tyrosine. Simulating the Figure 4B spectrum, which arises from $\beta, \beta-d_{2}$ Tyr substitution, is more problematical because the major radical signal cannot be distinguished well from a background signal such as that shown in Figure 3C or 3D. The simulation given in Figure 4B includes only $20 \%$ contribution from the protonated radical. The remaining $80 \%$ is simulated with parameters for the $\beta, \beta-d_{2}$ Tyr radical, although in reality about $30 \%$ of the intensity must arise from the unresolved background signal. The contribution of this background EPR signal is evident in the differences between simulation and experiment in the field range 330-335 $\mathrm{mT}$ (Figure 4A,C). Since the featureless radical EPR signals (see Figure 3) that overlap the resolved tyrosine signals are also centered at about $g=$ 2.004 , these background signals may also be of tyrosine origin. Further studies of the kinetics of cAOS radical formation, and studies of reaction under anaerobic conditions, are planned to examine the lifetime and relaxation of the tyrosyl radical and the origin of the second, featureless radical.

(b) Oxidation of cAOS Mutants. The mutation $\mathrm{Y} 193 \mathrm{H}$ of cAOS was chosen because, if Y193 were the radical site, not only the EPR signal might be changed but a histidine radical might be observed as well. Examples of histidine radicals in proteins are limited, probably because histidine has a higher oxidation potential $(\sim 1.2 \mathrm{~V}, \mathrm{pH} 7)$ than that of tyrosine or tryptophan $(0.75$ and $0.80 \mathrm{~V}$, respectively, $\mathrm{pH}$ 7) (39-41). However, only a small, featureless radical was observed when the Y193H mutant was oxidized (Figure 6). Two interpretations of the spectra in Figure 6 can be made. One is that no radical is formed at position Y193 when it is mutated to histidine and that the small signal observed may be the same as the second signal (as in Figure 3D) seen when native cAOS is oxidized. The other is that a short-lived histidine radical forms in $\mathrm{Y} 193 \mathrm{H}$ and the signal decays before the sample is frozen. In either case, residue 193 plays an important role in transfer of electrons to oxidized heme in cAOS. The $\mathrm{Y} 193 \mathrm{H}$ mutant is less stable under turnover conditions with nonnatural fatty acid hydroperoxide substrate than the native enzyme or the Y209F mutant. This was shown by determining how many molecules of $13 R$-HpODE are consumed before a molecule of cAOS is inactivated (Figure 5 and Table 3). Although initial rates of reaction of native and mutant cAOS with $13 R$-HpODE are quite similar, the reaction stops after consuming different amounts of hydroperoxide with native and mutant cAOS. These data suggest that one role of radical formation at Y193 is to stabilize coral AOS heme when abnormal interaction with a substrate occurs. In this regard, it is interesting to note that the heme iron in cAOS can return to ferric after a few turnovers of peracetic acid but not after exposure to a large excess of the oxidant.

(c) Selectivity in Tyrosine Oxidation. The amplitude and shape of the EPR radical signal in oxidized cAOS are essentially the same for addition of 1-10-fold molar excesses of peracetic acid, as long as the sample is frozen within 15 $\mathrm{s}$ of mixing. Formation of a radical at a single site is consistent with these results. However, if larger excesses of oxidant, or longer delays before freezing, are used, the characteristic tyrosine radical EPR signal decays and a narrower, featureless signal remains (Figure 3). It is possible that the site contributing the featureless radical signal is the source of the second electron that partially restores cAOS heme iron to the ferric state.

Possible Structural Similarity of cAOS and Catalases. Sequence alignments in some of the tyrosine-containing regions of cAOS and the catalases from $P$. mirabilis and Bos taurus are shown in Table 2. The site of tyrosine radical formation has been considered in both catalases. Distance measurements based on relaxation rates of catalase radicals place the radicals about $15-16 \AA$ from the iron of compound I. The F194Y mutant of the bacterial catalase forms a tyrosine radical on treatment with peracetic acid, whereas no radical is observed in this reaction of the native P. mirabilis enzyme. Y193 in coral AOS and Y214 in BLC occur in sequence positions equivalent to $\mathrm{F} 194 \mathrm{Y}$ in mutant PMC catalase. The radical site resulting from oxidation of BLC has not been demonstrated directly, but examination of side chain torsion angles of the tyrosines in the human erythrocyte catalase $\mathrm{X}$-ray structure (22) implicates Y360 as the radical site in erythrocyte catalase. By inference, then, the BLC radical is suggested to be at the equivalent Y369, not Y214 (21). Mutant F213Y of PMC has also been examined by others, and it does not form a radical (22). The closest tyrosine to this location that cAOS and BLC have in common is three residues earlier, Y209 in cAOS and Y235 in BLC. We examined this site by mutation of cAOS (Y209F), and a radical EPR signal identical to that of the native protein was obtained, eliminating Y209 in cAOS as the radical site. Although the sequence comparisons of cAOS and catalases have led to finding the site of the cAOS radical, the structure of cAOS has not been determined, so structural comparisons are speculative. The NADPH binding site of catalases is adjacent to the amino acid occupying a site equivalent to cAOS Y193. The tyrosine in BLC that is equivalent to cAOS Y193 is hydrogen bonded to two other residues: the phenolic oxygen is hydrogen bonded to an arginine, and the backbone hydrogen bonds to an asparagine. These hydrogen-bonding partners occur equivalently in the cAOS sequence at R181 and N137. If cAOS does have a structure similar to that of catalases $(21,43)$, the phenolic oxygen of Y193 would be held within $\sim 17 \AA$ of the heme iron by hydrogen bonds of Y193 to N137 and R181, and the R181-Y193 hydrogen bond to phenolic oxygen would facilitate deprotonation and the redox reaction observed. However, the EPR hyperfine 
splittings of the Y193 tyrosine radical in cAOS are distinctly different than those of mutant PMC (or of BLC), so the side chain torsion angles of Y193 in cAOS are also different from those in the one catalase that has been shown to form a radical at a sequentially equivalent position (PMC Y194).

Relation to Mechanism. Formation of a tyrosine radical in cAOS upon peracetic acid oxidation is best explained by heterolytic, peroxidase-type cleavage of the $\mathrm{O}-\mathrm{O}$ bond and reduction of compound I by Y193. These steps are confirmed by the stopped-flow optical results shown in Figure 2 . Heterolytic cleavage of a hydroperoxide contrasts with the proposed mechanism for reaction of cAOS with its normal substrate, which is thought to proceed by homolytic cleavage of the $\mathrm{O}-\mathrm{O}$ bond to form a substrate radical associated with $\mathrm{Fe}^{4+}-\mathrm{OH}(17)$. Fatty acid hydroperoxides are homolytically cleaved by cytochrome $\mathrm{P} 450$ 2B1 in a reaction proposed to pass through the same intermediates as the cAOS reaction (17). But this same P450 reacts with some aromatic hydroperoxides through a mixture of homolysis and heterolysis (18). Additionally, prostaglandin $\mathrm{H}$ synthase-2 (PGHS-2) gives a mixture of heterolytic and homolytic cleavage of alkyl hydroperoxide, while PGHS-1 is more specific (42). Although the mechanism proposed for coral AOS involves homolytic cleavage of the $8 R$-HpETE hydroperoxide substrate (17), it is not surprising that it can adopt the heterolytic mode of reaction with some other pseudosubstrates. With a small oxidant, or an improperly positioned hydroperoxide, water also may gain access to the substrate binding site and assist in developing negative charge on the internal oxygen of the hydroperoxide $\mathrm{O}-\mathrm{O}$ bond, as suggested for the $\mathrm{P} 450$ 2B1 heterolytic reaction (18).

Existence of cAOS as a fusion protein with a lipoxygenase suggests a need in coral for a highly selective pathway to a single isomeric allene oxide. Boutaud and Brash (7) examined the fatty acid specificity of the lipoxygenase domain to which cAOS is fused. The lipoxygenase reaction was most rapid with the substrate $22.6 \omega 3$, and somewhat slower with other C22 and C20 polyunsaturated fatty acids, while no reaction with $\mathrm{C} 18$ fatty acids was observed. These investigators also showed that cAOS inactivates after turning over fewer racemic hydroperoxide molecules than the number of preferred $R$-stereochemistry molecules required for inactivation (7). An interpretation of this observation is that the nonsubstrate $S$-stereoisomer is cleaved heterolytically, leading rapidly to inactivation. Although the coral lipoxygenase domain does not react with linoleic acid (7), we show here that $13 R$-HpODE, a linoleic acid product, does react with the cAOS domain, but the number of turnovers before cAOS is inactivated with this substrate is about $3 \%$ of the number observed (7) when the substrate is $8 R$-HPETE. The number of turnovers before inactivation is significantly smaller for the Y193H mutant, compared to native cAOS (Figure 5). This result suggests that formation of a radical at Y193 allows cAOS to recover more readily from an abortive, heterolytic interaction with hydroperoxide. As the Y193 radical is formed, oxidizing equivalents are drawn away from the heme. Together, these studies indicate that cAOS inactivates via heterolysis of substrates, especially when they do not fit the substrate binding site optimally. This mode of inactivation is likely a means of controlling specificity in the isomer of allene oxide formed when the fusion protein is functioning in the presence of lipoxygenases other than the one in the fusion protein or in the presence of air-oxidized lipids.

It is interesting to speculate that cAOS may actually use a readily formed radical at Y193 to activate polyunsaturated fatty acid substrates, in a manner similar to the cyclooxygenase pathway of prostaglandin synthase (42). There is normally low-level consumption by lipoxygenases of their hydroperoxide products, to keep the catalytic iron activated as ferric. As noted by others, a problem in maintaining ferric iron arises for the lipoxygenase fused to cAOS because the AOS domain rapidly converts hydroperoxides as they are formed (7). There is precedent in cyclooxygenase chemistry for the tyrosine radical of intermediate II to abstract hydrogen from polyunsaturated fatty acids (42). With cAOS, an unsaturated fatty acid could reduce the tyrosine radical and react further with oxygen to give a peroxyl radical. A peroxyl radical would readily oxidize the ferrous iron in the lipoxygenase. For this to be the case, it is important that it has been shown that the hydroperoxide requirement for activation of a lipoxygenase need not be specific to the stereochemistry of the product of that particular lipoxygenase (44). These possible cAOS radical reactions will be examined in further studies.

\section{ACKNOWLEDGMENT}

The help of A. Shriner, D. Paul, and G. Ottenberg with some of the cAOS expressions is gratefully acknowledged. We thank A. R. Brash for providing $8 R$-HpETE and the expression construct for cAOS, E. H. Oliw for providing $13 R$-HpODE, and H. Cooper for obtaining FT-ICR mass spectra.

\section{REFERENCES}

1. Brash, A. R. (1999) J. Biol. Chem. 274, 23679-23682.

2. Feussner, I., and Wasternack, C. (2002) Annu. Rev. Plant Biol. 53, 275-297.

3. Peters-Golden, M., and Brock, T. G. (2001) FEBS Lett. 487, $323-$ 326.

4. Brock, T. G., Maydanski, E., McNish, R. W., and Peters-Golden, M. (2001) J. Biol. Chem. 276, 35071-35077.

5. Froelich, J. E., Itoh, A., and Howe, G. A. (2001) Plant Physiol. $125,306-317$.

6. Koljak, R., Boutaud, O., Shieh, B.-H., Samuel, N., and Brash, A. R. (1997) Science 277, 1994-1996.

7. Boutaud, O., and Brash, A. R. (1999) J. Biol. Chem. 274, 3376433770 .

8. Abraham, B., Sono, M., Boutaud, O., Shriner, A., Dawson, J. H., Brash A. R., and Gaffney, B. J. (2001) Biochemistry 40, 22512259.

9. Enright, A. J., Illopoulos, I., Kyrpides, N. C., and Ouzouniz, C. A. (1999) Nature 402, 86-90.

10. Lau, S.-M. C., Harder, P. A., and O'Keefe, D. P. (1993) Biochemistry 32, 1945-1950.

11. Song, W.-C., Funk, C. D., and Brash, A. R. (1993) Proc. Natl. Acad. Sci. U.S.A. 90, 8519-8523.

12. Howe, G. A., Lee, G. I., Itoh, A., Li, L., and DeRocher, A. E. (2000) Plant Physiol. 125, 711-724.

13. Itoh, A., and Howe, G. A. (2001) J. Biol. Chem. 276, 3620-3627.

14. Wang, L.-H., Tsai, A.-L., and Hsu, P.-Y. (2001) J. Biol. Chem. 276, 14737-14743.

15. Hecker, M., and Ullrich, V. (1989) J. Biol. Chem. 276, 141-150.

16. Shyue, S.-K., Ruan, K.-H., Wang, L.-H., and Wu, K. K. (1997) J. Biol. Chem. 272, 3657-3662.

17. Chang, M. S., Boeglin, W. E., Guengerich, F. P., and Brash, A. R. (1996) Biochemistry 35, 464-471.

18. Kupfer, R., Liu, Y., Allentoff, A. J., and Thompson, J. A. (2001) Biochemistry 40, 11490-11501.

19. Benecky, M. J., Frew, J. E., Scowen, N., Jones, P., and Hoffman, B. M. (1993) Biochemistry 32, 11929-11933. 
20. Ivancich, A., Jouve, H. M., Sartor, B., and Gaillard, J. (1997) Biochemistry 36, 9356-9364.

21. Putnam, C. D., Arvai, A. S., Bourne, Y., and Tainer, J. A. (2000) J. Mol. Biol. 296, 295-309.

22. Olson, L. P., and Bruice, T. C. (1995) Biochemistry 34, 73357347.

23. Andreoletti, P., Gambarelli, S., Sainz, G., Stojanoff, V., White, C., Desfonds, G., Gagnon, J., Gaillard, J., and Jouve, H. M. (2001) Biochemistry 40, 13734-13743.

24. Studier, F. W., Rosenberg, A. H., Dunn, J. J., and Dubendorff, J. W. (1990) Methods Enzymol. 185, 60-89.

25. Senko, M. W., Hendrickson, C. L., Pasa-Tolic, L., Marto, J. A., White, F. M., Guan, S., and Marshall, A. G. (1996) Rapid Commun. Mass Spectrom. 10, 1824-1828.

26. Senko, M. W., Hendrickson, C. L., Emmett, M. R., Shi, S. D.-H., and Marshall, A. G. (1997) J. Am. Soc. Mass Spectrom. 8, 970976.

27. Jones, P., and Middlemiss, D. N. (1972) Biochem. J. 130, 411415.

28. Palcic, M. M., and Dunford, H. B. (1980) J. Biol. Chem. 255, $6128-6132$.

29. Blum, H., Chance, B., and Litchfield, W. J. (1978) Biochim. Biophys. Acta 534, 317-321.

30. Rodriguez-Lopez, J. N., Hernández-Ruiz, J., Garcia-Cánovas, F., Thorneley, R. N. F., Acosta, M., and Arnao, M. B. (1997) J. Biol. Chem. 272, 5469-5476.

31. Ivancich, A., Jouve, H. M., and Gaillard, J. (1996) J. Am. Chem. Soc. $118,12852-12853$

32. Hulsebosch, R. J., van den Brink, J. S., Nieuwenhuis, S. A. M., Gast, P., Raap, J., Lugtenburg, J., and Hoff, A. J. (1997) J. Am. Chem. Soc. 119, 8083-8094.

33. Dorlet, P., Seibold, S. A., Babcock, G. T., Gerfen, G., Smith, W. L., Tsai, A., and Un, S. (2002) Biochemistry 41, 6107-6114.
34. Karthein, R., Dietz, R., Nastainczyk, W., and Ruf, H. H. (1988) Eur. J. Biochem. 171, 313-320.

35. McConnell, H. M. (1957) Annu. Rev. Phys. Chem 8, 105-128.

36. Fessenden, R. W., and Schuler, R. H. (1963) J. Chem. Phys. 9, 2147-2195.

37. Tommos, C., Tang, X.-S., Warncke, K., Hoganson, C. W., Styring, S., McCracken, J, Diner, B. A., and Babcock, G. T. (1995) J. Am Chem. Soc. 117, 10325-10334.

38. Warncke, K., Babcock, G. T., and McCracken, J. (1996) J. Phys. Chem. 100, 4654-4661.

39. Brabec, V., and Mornstein, V. (1980) Biophys. Chem. 12, 159165 .

40. Lassmann, G., Eriksson, L. A., Himo, F., Lendzian, F., and Lubitz, W. (1999) J. Phys. Chem. 103, 1283-1290.

41. Gunther, M. R., Peters, J. A., and Sivaneri, M. K. (2002) J. Biol. Chem. 277, 9160-9166.

42. Smith, W. L., DeWitt, D. L., and Garavito, R. M. (2000) Annu. Rev. Biochem. 69 145-182.

43. Murthy, R. N., Reid, T. J., III, Sicignano, A., Tanaka, N., and Rossmann, M. G. (1981) J. Mol. Biol. 152, 465-499.

44. Chasteen, N. D., Grady, J. K., Skorey, K. I., Neden, K. J., Riendeau, D., and Percival, M. D. (1993) Biochemistry 32, 97639771.

45. Schroeder, W. A., Shelton, J. R., Shelton, J. B., Robberson, B., Apell, G., Fang, R. S., and Bonaventura, J. (1982) Arch. Biochem. Biophys. 214, 397-421.

46. Robinson, N. E. (2002) Proc. Natl. Acad. Sci. U.S.A. 99, $5283-5288$.

BI027427Y 